\section{Rethinking the Molecular Diagnostics for Methicillin-Resistant Staphylococcus aureus}

To the Editor-Infections due to methicillin-resistant Staphylococcus aureus (MRSA) strains are a common clinical problem that causes a high burden of disease and that often requires long-term treatment. ${ }^{1}$ MRSA rates vary in different countries, with rates being high in the United States, Middle and Far Eastern countries, and Southern Europe but relatively low in, for example, Northern Europe.

Encoded by different alleles of the mecA gene, an alternate penicillin-binding protein (PBP2a) is expressed in MRSA conferring resistance to $\beta$-lactam antibiotics (except ceftaroline and ceftobiprole). ${ }^{2}$ mecA is situated on a mobile genetic element named staphylococcal cassette chromosome mec (SCCmec). Currently, this mobile element occurs as 12 different types in $S$. aureus as well as in other clinically relevant staphylococci such as $S$. haemolyticus and S. epidermidis. ${ }^{3,4}$

In 2011, a divergent mecA homologue was discovered and subsequently designated mecC. mecC MRSA has been isolated from patients from Western and Central Europe ${ }^{3-5}$ It has also been detected in different domestic and wild animals including cattle, sheep, hedgehogs, storks, and fox. ${ }^{6}$ The reservoir of $m e c C$ strains is still unknown, although zoonotic transmission from cows or sheep is believed to play a major role.

The mecC gene was detected in various rare lineages of $S$. aureus in multilocus sequence type (MLST) clonal complexes (CC) 49, 130, 425, 599, and 1943. ${ }^{7}$ The amino acid identity between mecC- and mecA-encoded PBP2a is only $63 \%$. While the mecC gene itself does not mediate resistance to penicillin, it is frequently accompanied by the $\beta$-lactamase blaZ, which is part of the SCCmec XI element that does confer resistance to penicillin. ${ }^{8}$ Previous studies found that $m e c C$ also mediates resistance to oxacillin and cefoxitin. ${ }^{9}$

We report a 59-year-old male patient with a postthrombotic ulcer on his right lower leg measuring $3 \times 3 \mathrm{~cm}$. This patient had no other concomitant disease and no prior hospital admission. The bacterial culture of the wound swab was analysed with Vitek 2 (bioMérieux, Nürtingen, Germany) and broth microdilution according to EUCAST break points. The isolate was further analyzed using the $S$. aureus Genotyping Kit 2.0 (Alere Technologies, Jena, Germany).

After repetitive wound debridement, bacterial culture of a wound swab revealed an $S$. aureus isolate resistant to benzylpenicillin, oxacillin, and cefoxitin in the routine susceptibility test. Other tested antibiotics typically used for treatment of $S$. aureus (eg, glycopeptides and chinolones) were found to be susceptible.

The MRSA isolate carried the mecC gene was negative for Panton-Valentine leukocidin (PVL) and was assigned to the CC130 lineage. The isolate belonged to agr group III and capsule type 8 , and the mecC gene was present as a part of the SCCmec XI element together with the distinct blaZ. Despite the association of the mecC gene to zoonotic transmission, the patient reported no contact with livestock or any other potential animal source.

The patient was admitted to the hospital for treatment of the leg ulcer and was simultaneously MRSA sanitized according to the German guidelines on 5 consecutive days with an octenidin-containing wound compresses for 20 minutes per day, mupirocin nasal ointment, throat flushing with chlorhexidine, and skin washing with octenidin. After waiting for 3 days, control swabs were taken on 3 consecutive days from the nose, throat, skin, and wound. None of these 12 swabs were positive for $S$. aureus, which was interpreted as evidence for successful sanitization exclusively with topical treatment. During the following 2 years, all 10 swabs taken for diagnostic and screening purposes remained negative for $S$. aureus.

The prevalence of $m e c C$ in humans and animals is still low: the value reported in a meta-analysis was $0.009 \%$ (95\% confidence interval $=0.055-0.013 \%)$ of MRSA, ${ }^{10}$ which corresponds to an order of magnitude range of 1:100 to 1:1000 of typed MRSA. ${ }^{5}$ While mecC strains in humans do not appear to be particularly virulent, cohort studies are missing and case reports are sparse.

Currently, there is no recommendation on treatment of mecC MRSA. With regard to treatment options, minimum inhibitory concentrations (MICs) for methicillin and cefoxitin are usually lower than in conventional MRSA, but these compounds might not be effective. Penicillin is an inhibitor for the mecC gene product, but because $m e c C$ is accompanied by a specific penicillinase (also carried on the SCCmec XI element), monotherapy is not a treatment option. However, in vitro and in vivo models have shown an effective combination with a $\beta$-lactamase inhibitor. ${ }^{8}$ The patient was treated as an mecA MRSA case to overcome the risk of treatment failures in case of susceptible or intermediate oxacillin MICs.

The current setup to diagnose mecA/mecC of MRSA is multifaceted and time-consuming. Because of its divergent sequence, mecC and its gene product cannot be detected by all assays designed to identify mecA/PBP2a, ${ }^{4}$ and it has been suggested that $m e c C$ is largely underreported. The discrepancy between phenotypic resistance and mecA-negative molecular or proteinbased confirmatory tests might delay reporting to the physician and, thus, also delay the administration of an appropriate treatment. Hence, the discovery of mecC has resulted in the recognition of the need to redesign diagnostic tests.

In summary, clinicians and microbiologists should be aware of the changing facets of MRSA infections, particularly the emergence of mecC MRSA-conferred resistance against oxacillin. MRSA resistance warrants further investigation, especially in cases of discrepant testing results. Because the clinical experience is limited to case reports (probably due to the underreporting of mecC MRSA), a mecC MRSA infection should be treated as an mecA MRSA infection to avoid treatment failure.

\section{ACKNOWLEDGMENTS}

Financial support: This work was supported by a grant from the German Ministry of Education and Research (BMBF grant no. 01KI1204). 
Potential conflicts of interest: R.E. and S.M. are employees of Abbott Rapid Diagnostics, Alere Technologies GmbH, the company that manufactures the microarrays used for strain assignment.

\author{
Claudia Stein, PhD; ${ }^{1,4}$ \\ Jörg Tittelbach, $\mathrm{MD}^{2}$ \\ Stefan Monecke, MD; ${ }^{3,4}$ \\ Sebastian Weis, MD; ${ }^{1,5,6}$ \\ Oliwia Makarewicz, $\mathrm{PhD} ;{ }^{1,4}$ \\ Ralf Ehricht, $\mathrm{PhD} ;{ }^{3,4}$ \\ Mathias Pletz, $\mathrm{MD}^{1,4}$
}

Affiliations: 1. Center for Infectious Disease and Infection Control, Jena University Hospital, Jena, Germany; 2. Department of Dermatology, Jena University Hospital, Jena, Germany; 3. Abbott Rapid Diagnostics, Alere Technologies GmbH, Jena, Germany; 4. InfectoGnostics Research Campus; 5. Center for Sepsis Control and Care, Jena University Hospital, Jena, Germany; 6. Klinik für Anästhesiologie und Intensivmedizin, Jena University Hospital, Jena, Germany.

Address correspondence to Claudia Stein, Center for Infectious Disease and Infection Control, Jena University Hospital, Am Klinikum 1, Jena, Germany (claudia.stein@med.uni-jena.de).

Infect Control Hosp Epidemiol 2018;39:495-496

(C) 2018 by The Society for Healthcare Epidemiology of America. All rights reserved. 0899-823X/2018/3904-0022. DOI: 10.1017/ice.2018.17

\section{REFERENCES}

1. Weis S, Kaasch AJ, Rieg S, et al. Staphylococcus aureus bacteremia - a distinct entity. Deutsche medizinische Wochenschrift 2015;140:982-989.

2. Monecke S, Muller E, Schwarz S, et al. Rapid microarray-based identification of different mecA alleles in staphylococci. Antimicrob Agents Chemother 2012;56:5547-5554.

3. Garcia-Alvarez L, Holden MT, Lindsay H, et al. Meticillinresistant Staphylococcus aureus with a novel mecA homologue in human and bovine populations in the UK and Denmark: a descriptive study. Lancet Infect Dis 2011;11:595-603.

4. Shore AC, Deasy EC, Slickers P, et al. Detection of staphylococcal cassette chromosome mec type XI carrying highly divergent $m e c A, m e c I, m e c R 1, b l a Z$, and $c c r$ genes in human clinical isolates of clonal complex 130 methicillin-resistant Staphylococcus aureus. Antimicrob Agent Chemother 2011;55:3765-3773.

5. Paterson GK, Larsen AR, Robb A, et al. The newly described mecA homologue, mecALGA251, is present in methicillinresistant Staphylococcus aureus isolates from a diverse range of host species. J Antimicrob Chemother 2012;67:2809-2813.

6. Monecke S, Gavier-Widen D, Hotzel H, et al. Diversity of Staphylococcus aureus isolates in European wildlife. PLoS One 2016;11:e0168433.

7. Becker K, Ballhausen B, Köck R, et al. Methicillin resistance in Staphylococcus isolates: The "mec alphabet" with specific consideration of mecC, a mec homolog associated with zoonotic S. aureus lineages. Int J Med Microbiol 2014;304:794-804.

8. Ba X, Harrison EM, Lovering AL, et al. Old drugs to treat resistant bugs: methicillin-resistant Staphylococcus aureus isolates with mecC are susceptible to a combination of penicillin and clavulanic acid. Antimicrob Agents Chemother 2015;59: 7396-7404.

9. Ballhausen B, Kriegeskorte A, Schleimer N, et al. The mecA homolog mecC confers resistance against beta-lactams in
Staphylococcus aureus irrespective of the genetic strain background. Antimicrob Agents Chemother 2014;58:3791-3798.

10. Diaz R, Ramalheira E, Afreixo V, et al. Methicillin-resistant Staphylococcus aureus carrying the new mecC gene-a metaanalysis. Diagn Microbiol Infect Dis 2016;84:135-140.

\section{Outbreak of ST395 KPC-Producing Klebsiella pneumoniae in a Neonatal Intensive Care Unit in Palermo, Italy}

To the Editor-The spread of carbapenem-resistant Klebsiella pneumoniae (CR-Kp) is an emerging concern worldwide. Italy is a country endemic for Klebsiella pneumoniae carbapenemases (KPCs). ${ }^{1,2}$ KPC spread in neonatal intensive care units (NICUs) may represent a major safety issue for critical infants and a challenge in managing new admissions. ${ }^{3}$ In February 2014, we started an active surveillance program for colonization by multidrug-resistant organisms in 5 NICUs in Palermo, Italy. Inclusion criteria for patients were hospitalization for at least 48 hours and collection of at least 1 rectal swab. Samples were collected monthly in each NICU and cultured on selective media. A gram-negative bacterium resistant to at least 3 different groups of antimicrobial agents (eg, penicillins, cephalosporins, aminoglycosides, and/or carbapenems) was defined as a multidrug-resistant gram-negative (MDRGN). Colonization was defined as the isolation of an MDRGN without evidence of infection.

Swabs were inoculated onto MacConkey agar plates with 4 antibiotic disks containing gentamicin, amoxicillinclavulanic acid, meropenem, and ceftazidime and were then incubated. Colonies growing into each antibiotic inhibition halo were subcultured, and biochemical identification of isolated strains was performed using the API 20E system (BioMerieux, Marcy-l'Etoile, France). Antibiotic susceptibility testing was performed using a disk diffusion (DD) method on Mueller-Hinton agar plates with a panel of antimicrobials (ie, netilmicin, amoxicillin-clavulanic acid, cefotaxime, ceftazidime, ceftriaxone, cefepime, imipenem, aztreonam, and gentamicin) according to the Clinical and Laboratory Standards Institute guidelines. ${ }^{4}$ Colonies growing within meropenem and imipenem inhibition zones were subjected to E-test strips (BioMerieux, Marcy-l'Etoile, France) for minimum inhibitory concentration (MIC) determination. Antibiotic susceptibility testing and extended spectrum $\beta$-lactamase (ESBL) detection were performed using DD and a double-disk synergy test.

In December 2016, rectal swabs from 6 patients in $1 \mathrm{NICU}$ identified these patients as colonized by CR-Kp, and 8 isolates were collected. Klebsiella pneumoniae strains showing an extended drug-resistant phenotype were screened by 